\title{
Traduire
}

Revue française de la traduction

$231 \mid 2014$

A table !

\section{La transmission de l'étrangéité : traduire et retraduire Camus en suédois}

\section{Elisabeth Tegelberg}

\section{(2) OpenEdition}

Journals

Édition électronique

URL : http://journals.openedition.org/traduire/676

DOI : $10.4000 /$ traduire.676

ISSN : 2272-9992

Éditeur

Société française des traducteurs

Édition imprimée

Date de publication : 1 décembre 2014

Pagination : 97-110

ISSN : 0395-773X

\section{Référence électronique}

Elisabeth Tegelberg, «La transmission de l'étrangéité : traduire et retraduire Camus en suédois »,

Traduire [En ligne], 231 | 2014, mis en ligne le 01 décembre 2016, consulté le 01 mai 2019. URL : http:// journals.openedition.org/traduire/676; DOI : 10.4000/traduire.676 


\title{
La transmission de l'étrangéité : traduire et retraduire Camus en suédois
}

\author{
Elisabeth Tegelberg
}

\section{Introduction}

Le roman L'Étranger d'Albert Camus, l'expression littéraire la plus connue de la philosophie de l'absurde, a été traduit en suédois à deux reprises. La première traduction suédoise, faite par Sigfrid Lindström, a paru en 1946, c'est-à-dire quatre ans après l'édition originale française et un an avant la révision du texte d'origine par l'auteur. La retraduction, faite par Jan Stolpe, a paru en 2009, soit soixante-trois ans après la première traduction.

Vu la place de choix accordée à cette œuvre dans la littérature européenne du xxe siècle, il est plutôt étonnant qu'il y ait un décalage temporel aussi grand entre les deux traductions. Peutêtre cela est-il dû au fait que la première traduction avait été jugée satisfaisante, défendant bien sa place au cours des décennies passées, ou peut-être le livre a-t-il un peu perdu de son actualité après le succès extraordinaire qu'il avait connu dans les années cinquante et soixante ? Signalons aussi que beaucoup de francophones suédois, bien que moins nombreux d'année en année, ont su lire L'Étranger en version originale. Quoi qu'il en soit, que l'on ait misé sur une retraduction suédoise de ce roman confirme son statut de " classique " et montre qu'on a considéré comme important de le rendre accessible à de nouvelles générations de lecteurs suédois.

Un trait fondamental du livre est que la forme et le contenu n'en font qu'un, constituant pour ainsi dire une propre langue. Cette langue, ayant été qualifiée " d'écriture blanche " par Roland Barthes (1972 : 10), reflète le message central du livre, à savoir l'aliénation existentielle de son protagoniste Meursault. L'Étranger a fait époque en grande partie grâce à son style : neutre, dépouillé et dépourvu d'explications et d'ornements. Jean-Paul Sartre souligne qu'ı une phrase de L'Étranger c'est une île " (1947: 142), voulant dire par là que chaque phrase est une unité isolée sans relation avec les phrases environnantes. Ainsi, le style reflète la manière dont Meursault interprète notre existence : une série d'événements fragmentaires et aléatoires. 
En 1947, Albert Camus a apporté à son texte un certain nombre de modifications, rendant celui-ci encore plus neutre et plus laconique du point de vue stylistique, modifications qui soulignent l'importance qu'il attachait à la forme du roman. Les caractéristiques propres à celui-ci donnent lieu à de multiples possibilités d'interprétation et nous en font découvrir, à chaque relecture, de nouveaux aspects. C'est la raison pour laquelle il est primordial de garder en traduction ces caractéristiques, sinon la traduction risque de "rétrécir " le texte, de ne pas rendre la force suggestive qui en fait un roman d'exception.

L'Étranger peut donner l'impression trompeuse d'être facile à traduire : phrases courtes, majoritairement sous forme de principales, absence de constructions latinisantes ainsi qu'un ordre des mots simple, autrement dit un texte à première vue facilement accessible pour ce qui est de la langue. Cependant, le défi, c'est la langue en tant qu'expression de l'idée principale : l'aliénation existentielle du protagoniste. Le style du roman témoigne du détachement du narrateur-protagoniste. Le manque d'explications et de connecteurs susceptibles d'aider le lecteur à comprendre la manière de penser de Meursault contribue dans une large mesure à nous dépeindre le caractère énigmatique, parfois insaisissable, de celui-ci. Comme le dit Sartre : "L'Étranger n'est pas un livre qui explique " (1947: 127), ce qui laisse de temps à autre le lecteur désemparé devant les réactions, les mots et les gestes du protagoniste. Le manque d'engagement personnel propre à Meursault, se trouvant loin de l'attitude de la plupart des êtres humains face à la vie, fait partie de son "étrangéité ". Cette indifférence aux possibilités que lui offre la vie, se traduit, entre autres, par des phrases telles que "Ça m'était égal ", "Cela n'avait aucune importance ", "Cela ne signifiait rien ", "Cela ne voulait rien dire ", phrases récurrentes dans le livre, notamment dans des situations qui impliquent des choix considérés normalement comme importants dans la vie des hommes. Il s'agit là de phrases clé : résumant l'attitude du protagoniste envers la vie, elles sont, indirectement, à l'origine de sa condamnation à mort.

Cet article prend son point de départ dans l'hypothèse de la retraduction, lancée par Antoine Berman en 1990 (cf. Gambier 1994) et qui soutient que la retraduction d'une œuvre littéraire, dans une langue donnée, se distingue de la première traduction pour ce qui est de la fidélité au message du texte source. Selon cette hypothèse, le premier traducteur tend à aider le lecteur, à lui faciliter la lecture en adaptant la traduction au contexte de la culture cible ; tout en rendant ainsi la compréhension du texte plus aisée, il risque pourtant de "normaliser " le texte, le privant de ses caractéristiques essentielles, voire de ne pas transmettre son message. Le retraducteur, par contre, est censé être plus respectueux du texte d'origine, tentant d'être aussi fidèle que possible à son message. II peut en résulter un texte plus difficile à digérer, moins " accessible ", mais en même temps un texte qui transmet mieux les intentions de l'auteur. Cependant, l'Hypothèse de la retraduction est loin d'être incontestée (cf. Paloposki \& Koskinen 2004) et, même si elle s'avère sans aucun doute valable dans bien des cas, les exemples du cas inverse ne manquent pas. 
Nous nous proposons ici d'analyser un cas concret pour voir si l'Hypothèse de la retraduction peut s'y appliquer. L'Étranger est un livre particulièrement intéressant à étudier à la lumière de cette hypothèse, étant donné que, comme nous venons de le souligner, la langue y reflète de façon très conséquente le message, c'est-à-dire le sentiment d'étrangéité de Meursault face à la rencontre de l'homme avec le monde. Nous nous posons la question de savoir comment la notion d'" étrangéité " a été transmise dans les deux traductions suédoises et si les résultats obtenus sont conformes à l'Hypothèse de la retraduction. Si c'est le cas, cela ne veut pas dire, naturellement, que cette hypothèse soit généralement valide ; il s'agit plutôt d'analyser son éventuelle application à un cas spécifique. Nous ferons également entrer en ligne de compte la première traduction et quelques retraductions en anglais du même livre (Ballard 2000, Kaplansky 2004), mettant l'accent sur des différences et des ressemblances avec les traductions suédoises concernant la manière de transmettre le message de "l'étrangéité ".

Constatons d'emblée - ce qu'ont d'ailleurs fait plusieurs critiques lors de la parution de la retraduction suédoise - que celle-ci transmet mieux que la première traduction le style dépouillé de l'original. Se pose la question de savoir à quels égards les deux traductions en suédois se distinguent l'une de l'autre et dans quelle mesure les stratégies de traduction pratiquées ont sauvegardé le message du texte d'origine. (Nous avons consulté, cela va sans dire, la version modifiée par Camus en 1947 afin d'éviter d'adresser à la première traduction d'éventuels reproches injustifiés). Notre analyse portera sur les deux domaines du lexique et de la syntaxe. Nous laisserons de côté l'aspect temporel : le fait que certains mots suédois soient tombés hors d'usage durant les soixante années qui séparent les deux traductions, le texte demandant par là une certaine mise à jour lexicale, n'entre pas dans le cadre de l'Hypothèse de la retraduction.

\section{Lexique}

Dans le domaine du lexique, nous faisons une division en six groupes principaux que nous traiterons successivement.

\section{a) Traduction "lexicographique"}

En regardant les deux traductions suédoises de L'Étranger, on se rend vite compte d'un phénomène relevant du domaine lexical et souvent présent dans la première traduction, à savoir le recours à des traductions sémantiquement exhaustives des lexèmes français, traductions du genre qu'on retrouve fréquemment dans les dictionnaires. Cette tendance, par contre, ne se constate pas dans la retraduction. En voici deux exemples :

(1) II avait un canotier, un nœud papillon et une canne à main (34)

Han hade styv halmhatt, halsduk knuten som " fluga ", och käpp (Lindström, 30)

(styv " raide/dur ")

Han hade halmhatt, fluga och käpp (Stolpe, 28) 
(2) Et au bizarre petit bruit qui traversait la cloison, j'ai compris qu'il pleurait (61) Och av ett underligt svagt ljud som hördes genom den tunna mellanväggen förstod jag att han grät (Lindström, 51) (tunn « mince »)

Och av det underliga svaga ljudet som trängde genom väggen förstod jag att han grät (Stolpe, 51)

Les informations sémantiques "supplémentaires ", correctes certes, ne s'imposent pourtant pas dans les contextes donnés. Les précisions (styv, tunn) manquent ici d'importance, ayant cependant pour effet de rendre le texte plus riche en mots, moins concis, que l'original. Bien qu'il s'agisse d'une stratégie de traduction à première vue assez " inoffensive ", la fréquence de ce genre de précisions éloigne stylistiquement la première traduction du texte source, celuici, ne l'oublions pas, étant le reflet du caractère taciturne du protagoniste.

\section{b) Explicitation sémantique}

On peut, dans le texte de Lindström, repérer des ajouts qui, bien que sémantiquement impeccables, le rendent plus riche en mots sans y apporter réellement rien d'essentiel, sinon une note plutôt subjective, voire expressive. II ressort des exemples (3) et (4) que Stolpe a traduit littéralement les mots français, chose qui semble ici la solution la plus naturelle. Le retraducteur a donc su garder le caractère neutre de l'expression de l'original, réussissant ainsi à mieux refléter celui-ci. On pourrait pourtant objecter que le système lexical du suédois, à un plus haut degré que celui du français, tend à apporter à certains substantifs des précisions concrètes (cf. Tegelberg 2000) et que, de ce fait, les choix de Lindström sont bien fondés au seul point de vue de la langue cible. En effet, cela aurait pu être, me semble-t-il, un argument valable dans un autre contexte que celui dont il est question ici :

(3) [...] et la nuit était déjà noire au-dessus des arbres et des lampes (38)

[...] och natten redan började bli svart över trädkronorna och gatlyktorna (Lindström, 33)

(trädkronorna " les cimes d'arbres "; gatlyktorna litt. " les lampes de rues ")

[...] och natten redan var svart över träd och lyktor (Stolpe, 31)

(4) Le soleil était monté un peu plus dans le ciel : il commençait à chauffer mes pieds (21) Solen hade hunnit lite längre upp på himlen: mina fötter började bli varma av solstrålarna (Lindström, 19) (solstrålarna " les rayons du soleil ")

Solen hade stigit lite högre på himlen: den började värma mina fötter (Stolpe, 17)

\section{c) Addition lexicale}

Sur le plan sémantique, Lindström choisit à maintes reprises d'apporter à sa traduction des ajouts de son propre cru, ajouts qui, le plus souvent, sont en forme de propositions (5-7) ou de compléments de verbe ou de nom (8-10). Ces explicitations témoignent de prises de position subjectives de la part du protagoniste, traits qui ne se retrouvent pourtant ni dans l'original ni dans la retraduction de Stolpe. Encore une fois, le caractère aliéné du protagoniste s'affaiblit, fait dû à la tendance à "étoffer " le texte, à le " nourrir " lexicalement, faisant du anti-héros du 
roman un personnage plus bavard, plus ordinaire, donc d'un abord plus facile et qui ne nous repousse pas comme risque de le faire le Meursault de l'original. En plus de présenter un nombre considérable de mots qui ne figurent pas dans le texte source, et dont l'effet est de banaliser le récit, cette stratégie de traduction confère à la traduction un ton qui n'est pas celui de l'original. Ce genre d'ajouts, à première vue plutôt innocents si on les regarde séparément, n'en sont pas moins, vus dans leur ensemble, d'une portée stylistique non négligeable :

(5) [...] elle était plus heureuse ici (9)

[...] hade hon det bättre här, och trivdes bättre (Lindström, 9)

(och trivdes bättre " et s'y plaisait mieux ")

[...] hade hon det bättre här (Stolpe, 9)

(6) Je n'ai plus beaucoup fait attention à lui (16)

Sen gav jag inte så noga akt på vad han hade för sig (Lindström, 15)

(vad han hade för sig " ce qu'il fabriquait ")

Jag brydde mig inte så mycket om honom sen (Stolpe, 13)

(7) [...] et j'ai commencé à lui expliquer (9)

Och jag började förklara för honom hur det hängde ihop (Lindström, 9)

( hur det hängde ihop " comment cela se tenait ")

[...] och jag började förklara (Stolpe, 9)

(8) Auparavant, il ne la battait pas (48)

Förut hade han inte brukat klå henne ordentligt (Lindström, 41) (ordentligt " méthodiquement ")

Tidigare hade han inte slagit henne (Stolpe, 40)

(9) [...] nous avons entendu un bruit de semelles et de griffes sur les marches en bois de l'escalier (55)

[...] vi hörde ljudet av fotsteg och krafsande klor mot trätrappan (Lindström, 46) (krafsande " grattant ")

[...] vi hörde steg av fötter och tassar i trätrappan (Stolpe, 46)

(10) [...] perdu dans une nuée de chaleur (27)

[...] försvunnen i ett dis av dallrande solhetta (Lindström, 25) (dallrande "vibrant ")

[...] förlorad i ett moln av hetta (Stolpe, 22)

d) Itération lexicale

La façon d'" enrichir " le texte en matière lexicale constatée chez le premier traducteur se manifeste également dans sa prédilection pour l'itération lexicale, c'est-à-dire la coordination de deux ou de plusieurs mots, à sens plus ou moins identique, à l'aide de la conjonction och 
" et "; ; p. ex. " sûr et certain ", ce qui donne à l'énoncé un certain rythme en même temps qu'une certaine emphase. Encore une fois, c'est donc Lindström qui a recours à une solution plus riche en mots, faisant correspondre deux mots coordonnés par och à un terme unique du texte d'origine. Stolpe, par contre, toujours formellement fidèle à l'original, rend ces termes par un seul mot correspondant sémantiquement à celui du texte source :

(11) [...] le camion sautait sur les pavés inégaux du quai (40)

[...] lastbilen hoppade och skumpade på den ojämna stenläggningen på kajen (Lindström, 35) ( hoppade och skumpade " sautait et cahotait ")

[...] lastbilen skumpade på kajens ojämna stenläggning (Stolpe, 34)

(12) Alors nous sommes rentrés doucement (59)

Sen gick vi lugnt och beskedligt hem (Lindström, 49) (lugnt och beskedligt " doucement et sagement ")

Då gick vi sakta hem (Stolpe, 49)

(13) Parmi eux, les jeunes gens avaient des gestes plus décidés que d'habitude (37)

De ynglingar som jag såg i dessa skockar hade självsäkrare och bestämdare gester än vanligt (Lindström, 32) (självsäkrare och bestämdare " plus sûrs et plus décidés ")

Bland dem hade de unga männen mer bes/utsamma gester än vanligt (Stolpe, 30)

Les deux mots coordonnés ont un sens très similaire, au point d'être synonymes ( $c f$. Bendz 1965). Par leur soudure, ils en sont venus à constituer en quelque sorte une seule unité sémantique dont le rôle est de renforcer le sens et de conférer à l'énoncé un plus haut degré d'expressivité stylistique. Dans les cas cités (ainsi que dans bien d'autres relevés dans la première traduction), cette itération lexicale n'apporte rien au texte du point de vue sémantique, ayant pour seul effet de faire de l'original une version plus personnelle, parfois plus " pittoresque ". Or, ce n'est sans doute pas un hasard si Lindström a choisi de traduire certains termes par des lexèmes binaires, étant donné que, d'une façon générale, il arrive fréquemment qu'un mot de la langue source ne trouve pas en un seul mot de la langue cible son équivalent sémantique et stylistique ; à l'occasion, deux mots peuvent constituer la meilleure solution. Dans les cas cités, pourtant, l'itération lexicale ne fournit aucune information sémantique supplémentaire mais nuit plutôt au message du texte en rendant celui-ci plus prolixe, moins laconique que l'original.

\section{e) Renforcement expressif}

Un autre trait caractéristique de la première traduction et allant stylistiquement dans le même sens que ceux déjà mentionnés, c'est le recours à des mots plus "forts ", plus " expressifs ", que les termes correspondants du texte de Camus. Ce procédé donne à la traduction un ton plus subjectif, et au protagoniste un engagement qu'il ne ressent guère dans la version originale. Par conséquent, Meursault devient, dans cette traduction, plus amical, plus sociable, son 
aliénation existentielle n'ayant pas été proprement transmise. La retraduction, encore une fois, rend de manière sémantiquement et stylistiquement fidèle les mots figurant dans le texte d'origine, sans pour autant manquer aux habitudes linguistiques suédoises :

(14) J'ai dû avoir l'air fatigué (51)

Jag måtte ha sett uttröttad ut (Lindström, 44) (uttröttad " épuisé ")

Jag måste ha sett trött ut (Stolpe, 42)

(15) J'ai dit : "Bonsoir ", mais le vieux insultait toujours (43)

Jag sade "goddag", men gubben fortsatte att ösa ur sig skällsord (Lindström, 37)

(ösa ur sig skällsord " déverser des injures ")

Jag sa "Gokväll" men gubben fortsatte att skälla (Stolpe, 36)

(16) Il a frappé à la porte et on n’a plus rien entendu. II a frappé plus fort (56)

Han bultade på dörren, och det blev tyst därinne. Han bankade hårdare (Lindström, 47)

(bultade ... bankade " a tapé ... a cogné ")

Han knackade på dörren och sen hördes inget mer. Han knackade hårdare (Stolpe, 47)

(17) II a ajouté que Raymond devrait avoir honte d'être soûl au point de trembler comme il le faisait (57)

Han tillade att Raymond borde skämmas över att vara så full att han skalv $i$ alla leder, som han gjorde (Lindström, 48) (skalv i alla leder " tremblait de tous ses membres ")

Han tillade att Raymond borde skämmas över att han var så berusad att han skakade som han gjorde (Stolpe, 48)

\section{f) Modalisation}

Un cas particulier d'ajout, très fréquent dans la première traduction mais presque totalement absent dans la retraduction, ce sont les mots et expressions dont la fonction est de " modaliser "l'énoncé, d'en atténuer la force énonciative. En introduisant dans son texte de tels " modalisateurs ", le traducteur en facilite la lecture ainsi que la compréhension du récit, rendu plus cohérent grâce à ceux-ci. Ils sont aussi d'intérêt à cause de la " personnalisation " du style qu'ils comportent, impliquant, souvent, des prises de position de la part de celui qui s'en sert. II est évident que Lindström, en recourant à des modalisateurs, tend à interpréter le texte de Camus, ou à expliquer ce qui ne doit pas être expliqué puisque l'auteur ne le fait pas, plutôt que de le traduire. Voilà qui reste problématique vu que chaque phrase de L'Étranger, étant « une île " (Sartre 1947 : 142), se suffit à elle-même et que c'est précisément l'absence d'explications, caractéristique de la manière de s'exprimer de Meursault, qui reflète son attitude envers la vie. Les exemples (18-24) illustrent la manière dont Lindström pratique la modalisation :

(18) [...] des mâts qui dansaient sur l'horizon (40)

[...] master som tycktes dansa mot horisonten (Lindström, 34) (tycktes dansa " semblaient danser ")

[...] master som dansade mot horisonten (Stolpe, 34) 
(19) J'ai couru pour ne pas manquer le départ (8)

Jag måste springa för att inte komma för sent till bussen (Lindström, 8) (måste springa « ai dû courir ")

Jag sprang för att hinna med bussen (Stolpe, 8)

(20) Mais je voulais vous en informer (11)

Men jag ansåg att jag borde underrätta er om det (Lindström, 11) (jag ansåg att "j'ai pensé que ")

Men jag ville tala om det för er (Stolpe, 10)

(21) [...] et tout aura revêtu une allure plus officielle (8)

[...] och det hela har fått en så att säga mer officiell karaktär (Lindström, 7)

(så att säga " pour ainsi dire ")

[...] och allt blir mer officiellt (Stolpe, 7)

(22) Bien entendu, cela ne m'empêche pas de comprendre tout de même mon patron (30) Men märk väl, detta hindrar alls inte att jag i alla fall förstår chefens känslor (Lindström, 27) (men « mais "; alls " du tout ")

Det hindrar förstås ändå inte att jag kan förstå chefen (Stolpe, 25)

(23) Un dernier mot (11)

Och nu bara ett ord till (Lindström, 10) (nu bara " maintenant seulement ")

Och sist (Stolpe, 10)

(24) J'ai pensé alors que je n'aurais pas dû lui dire cela (7)

Då tänkte jag att jag kanske inte borde ha sagt så (Lindström, 7) (kanske " peut-être ")

Då tänkte jag att det där borde jag inte ha sagt (Stolpe, 7)

Au niveau lexical, Lindström va nettement, on l'a constaté, à la rencontre du lecteur (étant donc cibliste), parce qu'il fait de sa traduction un texte plus accessible et qui offre moins de résistance intellectuelle et émotionnelle, tout en lui faisant perdre une partie de sa force suggestive et de son caractère ambigu. De son côté, Stolpe va à la rencontre de l'auteur (étant donc sourcier), parce qu'il transmet fidèlement au lecteur les caractéristiques lexicales et stylistiques du récit et, par là, son message essentiel : l'aliénation propre au protagoniste (sur ces deux attitudes traductives à l'égard de l'auteur et du lecteur, voir Schleiermacher 1963 et Mounin 1994).

\section{Syntaxe}

Les différences relatives à la fidélité au message du texte d'origine, relevées dans les deux traductions impliquées, ne se limitent pas aux domaines lexical et sémantique mais se mani- 
festent aussi de façon très claire dans celui de la syntaxe. Ici, on constate, à l'instar de ce qui est le cas pour le lexique, que le retraducteur respecte plus scrupuleusement que ne le fait le premier traducteur les structures syntaxiques de l'original. Que Stolpe réussisse à être fidèle aux caractéristiques structurales du texte français, sans le faire au détriment de la lisibilité en langue cible, est d'autant plus remarquable que le suédois se distingue sur bien des points du français pour ce qui est des structures syntaxiques. Il est donc loin d'être évident de faire une traduction reflétant syntaxiquement le texte source. Ainsi, le français se sert à un plus haut degré que le suédois de constructions nominales - surtout en forme de syntagmes prépositionnels - là où le suédois préfère, de façon générale, les propositions subordonnées, en particulier des circonstancielles temporelles (1-3), mais aussi des relatives (4), des complétives (5) et des interrogatives (6) (cf. Eriksson 1993). Cela ne signifie pas, bien entendu, que les constructions nominales soient toujours impossibles en suédois - ni que le français se serve peu de subordonnées -, seulement qu'elles y sont moins courantes et, dans bien des cas, impossibles ou idiomatiquement inacceptables.

Stolpe a réussi à rendre les constructions françaises par des tournures suédoises analogues. Lindström, de son côté, tout en restant fidèle au système syntaxique du suédois, les a rendues par des subordonnées, se conformant par-là à la tendance générale du suédois :

(1) Après l'enterrement, au contraire, ce sera une affaire classée (8)

Men när begravningen är över, då är saken utagerad (Lindström, 7) (när begravningen är över " quand l'enterrement sera fini ")

Efter begravningen är saken tvärtom utagerad (Stolpe, 7) (efter begravningen " après l'enterrement ")

(2) Au bout de quelques mois, elle aurait pleuré (10)

När hon hade varit där några månader, skulle hon ha gråtit (Lindström, 9) (när hon hade varit där några månader " quand elle y aurait passé quelques mois ")

Efter några månader skulle hon ha gråtit (Stolpe, 9) (efter några månader " au bout de quelques mois ")

(3) Dans l'escalier, il m'a expliqué (10)

När vi var i trappan, förklarade han för mig (Lindström, 10) (när vi var i trappan « quand nous nous trouvions dans l'escalier ")

I trappan förklarade han (Stolpe, 9) (i trappan " dans l'escalier ")

(4) [...] elle avait des amis, des gens de son âge (10)

[...] här hade hon vänner, folk som var jämngamla med henne (Lindström, 9) (som var jämngamla med henne "qui avaient le même âge qu'elle ")

[...] hon hade vänner, människor $i$ hennes egen ålder (Stolpe, 9) (i hennes egen ålder « de son [propre] âge ") 
(5) [...] on ne s'est pas fait à l'idée que déjà il faut courir derrière le corbillard (14)

[...] man hinner inte väl sätta sig in i att den anhörige är död förrän det är dags att springa efter likvagnen (Lindström, 13) (att den anhörige är död " que le parent est mort ") [...] man hinner knappt vänja sig vid tanken förrän man ska springa bakom likvagnen (Stolpe, 12) (tanken "l'idée ")

(6) [...] parce que je ne savais pas le chiffre exact (26)

[...] därför att jag inte visste hur gammal han var (Lindström, 24) (hur gammal han var « quel âge il avait ")

[...] för jag visste inte den exakta siffran (Stolpe, 21) (den exakta siffran « le chiffre exact »)

Les solutions de Lindström ne manquent donc pas de respect pour le texte d'origine, ayant tenu compte des différences structurales qui existent entre les deux langues. Cependant, dans ce cas-ci, il s'avère tout à fait possible de rendre presque mot à mot les structures de l'original sans que leur caractère idiomatique soit mis en cause. L'avantage d'une traduction littérale est ici de sauvegarder le laconisme de l'écriture camusienne, de respecter cette "stérilité magnifique " dont parle Sartre (1947 : 129) et qui reflète si bien le message existentiel, l'aliénation de Meursault. On peut, certes, objecter, en regardant les exemples donnés ci-dessus, qu'opter pour une subordonnée au lieu de recourir à une construction nominale ne semble pas trop dramatique du point de vue du message ; pourtant, même si chaque cas particulier n'est pas, en effet, de très grande importance, c'est la régularité avec laquelle Lindström choisit cette stratégie qui ne manque pas d'avoir des conséquences négatives pour le profil du texte traduit. On traduit toujours, rappelons-le, un texte, non pas des mots.

\section{Traductions anglaises}

II semble naturel, à titre comparatif, de dire quelques mots sur les traductions anglaises de L'Étranger. Celui-ci a été traduit en anglais à plusieurs reprises ( cf. les textes cités dans la bibliographie), fait qui n'a pas manqué de donner lieu à des études comparatives sur ces textes (cf. Ballard 2000 et Kaplansky 2004). Qu'il y ait plusieurs retraductions anglaises n'est guère étonnant, vu que ce roman marque une date importante dans l'histoire littéraire européenne du xxe siècle. Bien connu dans le monde anglo-saxon, il est, comme en Suède, un des livres de cours obligatoires aux départements de français des universités américaines et anglaises.

Dans son article, Jonathan Kaplansky (2004) compare quatre traductions en anglais de L'Étranger. II y analyse la manière dont les différentes stratégies des traducteurs ont influé sur le contenu, sur le profil linguistique, sur la " voix " du texte traduit. Les plus grandes déviations par rapport au texte de Camus se retrouvent dans la première traduction, faite par Stuart Gilbert et parue en 1946. Celui-ci, à l'instar de Sigfrid Lindström, entretient une relation plus libre que les retraducteurs avec le texte d'origine. Michel Ballard (2000) a fait une comparaison 
entre trois des traductions anglaises et arrive à peu près à la même conclusion que Kaplansky. Ces deux chercheurs relèvent entre autres le fait que Gilbert établit des " connecting links ", explicitant ainsi des liens de cause à effet autrement que ne le fait le texte de Camus, lequel, comme le dit Sartre, "n'est pas un livre qui explique " (1947: 127). La traduction de Gilbert se signale aussi par son recours aux modalizers (Kaplansky).

Ballard, aussi bien que Kaplansky, constatent que la traduction de Gilbert est plus riche en mots (wordier; Kaplansky [2004 : 189]) que l'original, ce qui l'éloigne de l'" écriture blanche " qui, selon Barthes (1972: 10), lui est propre. Les ajouts faits par ce traducteur rendent le texte plus personnel, si bien qu'il se trouve parfois assez loin de l'objectivité caractéristique de la langue du protagoniste. Ainsi, l'» étrangéité " de celui-ci devient moins prononcée dans cette première traduction que dans les retraductions anglaises, en particulier dans celle de Joseph Laredo (1982), qui respecte scrupuleusement le style de Camus au point même de présenter à l'occasion, selon Kaplansky (2004 : 191), un texte pas tout à fait idiomatique en anglais.

Gilbert s'est également servi du procédé appelé " étoffement " par Kaplansky (2004 : 192)(1). Par ce terme, celui-ci entend que le traducteur " orne " ou "brode " le texte qu'il traduit, donnant sa propre interprétation de l'original plutôt que de traduire ce qui y est exprimé réellement. Voilà qui ressort par exemple de la phrase suivante, où hardly conscious, hope et some agreeable surprise ne trouvent d'équivalent ni dans le texte de Camus, ni dans la traduction de Laredo :

(1) En réalité, je n'étais pas réellement en prison les premiers jours : j'attendais vaguement quelque événement nouveau (Camus ; cit. Kaplansky 2004 : 192)

(1a) In actual fact I wasn't actually in prison the first few days: I was vaguely waiting for something to happen (Laredo ; cit. ibid.)

(1b) In point of fact, during those early days, I was hardly conscious of being in prison; I had always a vague hope that something would turn up, some agreeable surprise (Gilbert ; cit. ibid.)

Meursault nous apparaît, dans la première traduction, plus prolixe, plus engagé que la création de Camus, souligne Kaplansky. L'image du protagoniste que nous présente cette première version anglaise est donc tout autre que celle que donne le texte d'origine. Rappelons à ce propos encore une fois les mots de Sartre sur L'Étranger : " [s] on roman veut être d'une stérilité magnifique " (1947 : 129), stérilité qui ne caractérise pourtant pas au même degré la traduction de Gilbert. Ce traducteur, il est vrai, a rendu son texte plus accessible aux lecteurs anglais, plus facile à " digérer " mais en même temps moins ouvert à des interprétations

(1) Qui reprend J.-P. Vinay et J. Darbelnet, Stylistique comparée du français et de l'anglais (Paris, Didier, 1958), p. 109. 
possibles, ayant à plusieurs reprises banalisé la technique narrative de l'auteur, technique qui d'une façon si brillante reflète l'aliénation existentielle du protagoniste.

On ne peut pas ne pas être frappé par les ressemblances stratégiques entre les premières traductions suédoise et anglaise de L'Étranger. Celles-ci sont, on l'a vu, moins fidèles, plus "désinvoltes ", par rapport au texte d'origine que ne le sont les retraductions. À cause des stratégies pratiquées par Sigfrid Lindström et Stuart Gilbert, Meursault devient plus aimable, plus bavard, donc moins aliéné, moins " étranger ", que dans le texte de Camus.

Les deux premiers traducteurs dont il est question ici, prenant un grand nombre de libertés sémantiques et stylistiques vis-à-vis de l'original, vont à la rencontre du lecteur, lui facilitant la lecture de ce texte, au fond très complexe. Cependant, en même temps, ils le privent en partie de son message philosophique et de sa force suggestive qui en ont fait un "classique " de la littérature française. Les libertés que se sont accordées Sigfrid Lindström et Stuart Gilbert sont, dans le cas spécifique qu'est L'Étranger, particulièrement malheureuses vu le lien étroit qui y existe entre forme et contenu : le caractère "étranger " du protagoniste trouve sa réplique dans le style neutre, dépouillé et lapidaire du récit. Comme nous l'avons vu, toute déviation sémantique et stylistique de la part du traducteur risque d'être lourde de conséquences.

En suédois aussi bien qu'en anglais, les retraductions, par leur respect pour la manière de s'exprimer de Meursault, réussissent mieux que les premières traductions à lui faire garder le trait primordial de sa personnalité, son " étrangéité ".

\section{Conclusion}

Mises ensemble, les différences relevées entre les deux traductions suédoises influencent de manière décisive le profil du texte de Camus. Dans chaque cas particulier, Jan Stolpe se trouve, on l'a vu, plus près que Sigfrid Lindström du texte d'origine, captivant de façon convaincante le style de celui-ci. À mon avis, on peut même prétendre que la retraduction se trouve extrêmement près du texte français. Le retraducteur est resté scrupuleusement fidèle à l'auteur, ayant su, de manière conséquente, éviter de "normaliser "Meursault et de le rendre, à travers ses mots, plus engagé qu'il ne l'est.

II me semble que Jan Stolpe, dans sa traduction, a fait preuve d'une grande sensibilité, d'une compréhension profonde des idées philosophiques mises en valeur par l'auteur, ayant su capter le ton et le rythme tout particuliers de L'Étranger, sa " voix " tout court. Il a su recréer la langue et le style et transmettre autant que faire se peut le message de l'original (les trois formant en réalité un tout). Cette retraduction a, à mon avis, de bonnes possibilités de devenir un classique dans le domaine de la retraduction littéraire du français vers le suédois.

Sigfrid Lindström, à en juger par sa traduction, ne semble pas avoir fait siennes, au même degré, les caractéristiques essentielles de L'Étranger. N'oublions pas, cependant, la situation 
favorable où se trouve le retraducteur par rapport à celle du premier traducteur d'une œuvre littéraire (cf. Tegelberg 2011) : il est incontestable que, de nos jours, nous savons beaucoup plus sur le roman de Camus et sur la philosophie qui en est à l'origine que ce n'était le cas en 1946 où le livre a été traduit en suédois pour la première fois. Le point de départ de Sigfrid Lindström est, par conséquent, très différent de celui de Jan Stolpe et sa traduction, qui est loin d'être mauvaise, doit être considérée à la lumière non seulement de la personnalité du traducteur mais aussi de l'époque où la traduction a été faite.

La comparaison des deux traductions en suédois de L'Étranger d'Albert Camus a donc eu pour résultat de corroborer l'Hypothèse de la retraduction. Le résultat d'un coup d'œil comparatif jeté sur les traductions en anglais du même roman va dans le même sens. Ces résultats, si probants soient-ils dans le cas particulier de L'Étranger, ne suffisent pourtant pas à donner à cette hypothèse une quelconque validité générale.

elisabeth.tegelberg@sprak.gu.se

Elisabeth Tegelberg est maitre de conférences au Département de langues et littératures de I'Université de Göteborg (Suède). Ses recherches portent sur trois domaines principaux : traductologie (en particulier les problèmes relatifs à la traduction du suédois vers le français), relations culturelles francosuédoises et didactique des langues. Elle a publié un grand nombre d'articles et plusieurs livres dans ces trois domaines de recherche. Depuis quelques années, elle s'intéresse particulièrement aux questions liées à la retraduction littéraire.

\section{Bibliographie}

Ballard, Michel. 2000. "In Search of the Foreign: A Study of Three English Translations of Camus' L'Étranger ". In On Translating French Literature and Film II, ed. by Miriam Salama-Carr, 19-38, Atlanta (États-Unis) : Rodopi.

Barthes, Roland. 1972. Le degré zéro de l'écriture suivi de Nouveaux essais critiques. Paris : Seuil. 187 p.

Bendz, Gerhard. 1965. Ordpar. Stockholm : Norstedt. 147 p.

Berman, Antoine. 1990. "La Retraduction comme espace de traduction ". In Retraduire, édité par Paul Bensimon, 1-7, Paris : Presses de la Sorbonne Nouvelle.

Eriksson, Olof. 1993. La Phrase française. Essai d'un inventaire de ses constituants syntaxiques. Göteborg (Suède) : Acta Universitatis Gothoburgensis. 408 p.

Gambier, Yves. 1994. "La Retraduction, retour et détour ", Meta 39 (3) : 413-417. 
Kaplansky, Jonathan. 2004. "Outside The Stranger? English Retranslations of Camus' L'Étranger". In Pourquoi donc retraduire ?, édité par Paul Bensimon, 187-198, Paris : Presses de la Sorbonne Nouvelle.

Mounin, Georges. 1994 [1955]. Les Belles Infidèles. Lille (France) : Presses Universitaires de Lille. 109 p.

Paloposki, Outi et Kajsa Koskinen. 2004. "A thousand and one translations". In Claims, Changes and Challenges in Translation Studies, ed. by G. Hansen, K. Malmkjær \& D. Gile. Selected Contributions from the EST Congress, Copenhagen 2001, 27-37, Amsterdam : John Benjamins.

Sartre, Jean-Paul. 1975 [1947]. "Explication de L'Étranger ", Critiques littéraires (Situations, I). Paris : Gallimard.

Schleiermacher, Friedrich. 1963 [1816]. "Über die verschiedenen Methoden des Übersetzens ". In Das Problem des Übersetzens, édité par Hans Joachim Störig, Darmstadt (Allemagne) : Wissenschaftliche Buchgesellschaft, 38-70.

Tegelberg, Elisabeth. 2000. Från svenska till franska. Kontrastiv lexikologi i praktiken. Lund (Suède) : Studentlitteratur. $247 \mathrm{p}$.

Tegelberg, Elisabeth. 2011. "La retraduction littéraire - quand et pourquoi ? ", Babel 57 (4) : 452-471.

\section{Textes}

Camus, Albert. 1957 [1942]. L'Étranger. Paris : Gallimard ("Pléiade ").

- 1946. Främlingen, trad. Sigfrid Lindström. Stockholm, Bonnier.

- 2009. Främlingen, trad. Jan Stolpe. Stockholm, Bonnier.

- 1954 [1946]. The Stranger, trad. Stuart Gilbert. New York (États-Unis) : Vintage.

- 1982. The Stranger, trad. Kate Griffith. Washington D.C. : University Press of America.

- 1983 [1982]. The Outsider, trad. Joseph Laredo. London : Penguin.

- 1989 [1988]. The Stranger, trad. Matthew Ward. New York (États-Unis) : Vintage. 